\title{
A case-control study on red meat consumption and risk of stroke among a group of Iranian adults
}

\author{
Parvane Saneei ${ }^{1}$, Mohammad Saadatnia ${ }^{2}$, Forough Shakeri ${ }^{3}$, Masumeh Beykverdi $^{1}$, \\ Ammar Hassanzadeh Keshteli ${ }^{4,5}$ and Ahmad Esmaillzadeh ${ }^{1, *}$ \\ ${ }^{1}$ Food Security Research Center, Department of Community Nutrition, School of Nutrition and Food Science, Isfahan \\ University of Medical Sciences, Isfahan, PO Box 81745-151, Islamic Republic of Iran: ${ }^{2}$ Isfahan Neuroscience \\ Research Center, Isfahan University of Medical Sciences, Isfahan, Islamic Republic of Iran: ${ }^{3}$ Medical Students' \\ Research Center, Isfahan University of Medical Sciences, Isfahan, Islamic Republic of Iran: ${ }^{4}$ Integrative Functional \\ Gastroenterology Research Center, Isfahan University of Medical Sciences, Isfahan, Islamic Republic of Iran: \\ ${ }^{5}$ Department of Medicine, University of Alberta, Edmonton, Alberta, Canada
}

Submitted 8 October 2013: Final revision received 16 April 2014: Accepted 6 May 2014: First published online 13 June 2014

\begin{abstract}
Objective: We aimed to examine the association between red meat consumption and stroke in a group of Iranian adults.

Design: A hospital-based case-control study.

Setting: The study included stroke patients and hospital-based controls. Usual dietary intakes of participants were assessed by means of a validated 168-item semi-quantitative FFQ. Total red meat consumption was calculated by summing up the consumption of red, processed and visceral meats.

Subjects: One hundred and ninety-five cases were stroke patients hospitalized in the neurology ward and 195 controls were recruited from patients hospitalized in other wards with no history of cerebrovascular diseases or neurological disorders. Results: Participants with stroke were older, more likely to be male and less likely to be obese. Individuals in the highest tertile of red meat intake were $119 \%$ more likely to have stroke (OR $=2 \cdot 19 ; 95 \%$ CI 1.33 , 3.60) compared with those in the lowest tertile. After controlling for age, sex and total energy intake, the association between red meat consumption and stroke was strengthened (OR $=2 \cdot 72 ; 95 \% \mathrm{CI}$ $1 \cdot 53,4 \cdot 83)$. This association remained significant even after further controlling for physical activity and smoking as well as dietary intakes. Additional adjustments for BMI, diabetes, hypertension and hyperlipidaemia did not influence the association significantly (OR $=2 \cdot 51 ; 95 \%$ CI $1 \cdot 19,5 \cdot 09)$.

Conclusions: Consumption of red meat was associated with greater odds of having stroke in a group of Iranian adults.
\end{abstract}

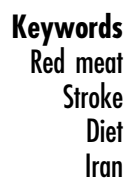

Stroke is a leading cause of death and disability worldwide and will impose a great burden on health-care systems in future decades ${ }^{(1,2)}$. Despite numerous epidemiological data on stroke prevalence in the USA and Western countries $^{(1,2)}$, little information is available from population-based studies in developing countries ${ }^{(3)}$. Findings from prior investigations in Iran have revealed that the incidence of stroke is greater than in most Western countries, with stroke occurring at younger ages and ischaemic stroke being more prevalent than other subtypes ${ }^{(4)}$. Therefore, primary prevention of stroke is of great priority in these countries.

Dietary intake of several nutrients and foods has previously been linked to stroke; therefore, dietary modification may be a crucial way to reduce risk of stroke. Meat is widely used in the diet globally and is a main source of protein and some other nutrients in most diets. Earlier evidence from epidemiological studies has indicated that high consumption of red meat, particularly processed meat, might contribute to cancer ${ }^{(5)}, \mathrm{CHD}^{(6)}$, type 2 diabetes $^{(7)}$ and the metabolic syndrome ${ }^{(8)}$. High intake of red meat is also significantly associated with the prevalence of hypertension $^{(9)}$ and dyslipidaemia ${ }^{(8)}$, two well-known risk factors for stroke.

Epidemiological data on the relationship between red meat consumption and stroke are sparse and inconsistent ${ }^{(10-13)}$. A published meta-analysis of cohort studies reported no significant association between consumption of red and processed meats and stroke ${ }^{(14)}$; whereas findings from another recent meta-analysis of five cohort studies revealed that high red meat intake is associated with a $9 \%$ increase in the risk of stroke ${ }^{(15)}$. 
Each of these two meta-analyses included only one investigation conducted in an Asian population, which may not reflect the associations that typically exist in the Middle Eastern region. In a prospective cohort study of 51683 Japanese adults ${ }^{(16)}$ that was not included in the previously mentioned meta-analyses ${ }^{(14,15)}$, the authors found no link between moderate consumption of meat (including beef, pork, poultry, liver and processed meat) and mortality from stroke. Therefore, further evidence is required to clarify the possible relationships.

Almost all previous studies on the relationship between red meat intake and stroke have been done in developed countries, where dietary intakes of red and processed meats are high. Data in this regard are lacking from developing nations, in particular from the Middle Eastern populations whose dietary intakes of meat might be low to moderate. Moreover, the major sources of red meat intake in these countries are different from those developed countries, where pork is a major source of red meat intake. Some studies have indicated that the source of red meat intake may contribute to the association between red meat intake and CVD events ${ }^{(17)}$. Furthermore, the dietary intakes of Iranians are greatly different from those in Western countries ${ }^{(18)}$ and investigating the red meat-stroke association in this diversified mixed diet might provide additional information. Since findings from epidemiological studies are better replicated in a different range of dietary intakes in different populations, we hypothesized that red meat intake might contribute to the increasing prevalence of stroke among the Iranian population. Given the paucity of data on diet-disease relationships in the understudied population of the Middle East, we examined the association between red meat consumption and stroke in a group of Iranian adults.

\section{Materials and methods}

\section{Participants}

The current hospital-based case-control study included 195 stroke patients and 195 hospital controls. All participants were recruited from Alzahra University Hospital, Isfahan, Iran. Data about all cases and controls in the study were collected between January and December 2008. Data on each pair of cases and controls were collected at the same time. Cases were selected through a convenience non-random sampling procedure. They were stroke patients who were hospitalized in the neurology ward of Alzahra University Hospital. Controls were selected from hospitalized patients in orthopaedic or surgical wards of this centre. They were without a prior history of cerebrovascular accident or any neurological disorders and were not hospitalized owing to vascular-related conditions. The participation rate in the study was $100 \%$ for cases and $93 \%$ for controls. Written informed consent was obtained from each participant.

\section{Assessment of dietary intake}

A validated 168-item FFQ was used to assess the intakes of foods usually consumed by the participants over the previous year ${ }^{(19)}$. All questionnaires were administered by trained dietitians. The quantity of the food consumed was assessed by asking a standard common portion size. As brain damage or impaired memory is the most common sequela of stroke and recalls of dietary intakes from these patients might be distorted, we asked their family members to help complete the FFQ. The reported frequency as well as the portion size was double checked with the person responsible for cooking at home. The daily frequency of foods was calculated by dividing weekly frequency consumption by seven and monthly frequency by thirty, and the average daily intake of foods and beverages was calculated by multiplying the portion size by the daily frequency of consumption. Total energy intake was calculated by summing up energy intakes from all foods. Total red meat consumption was calculated by summing up the consumption of red meat (beef, veal, mutton, lamb), processed meat (sausages, hamburgers, hot dogs) and visceral meats (lamb's liver, kidney, heart).

\section{Assessment of stroke}

Patients aged $>45$ years with first ever symptomatic acute stroke diagnosed by a neurologist and confirmed by brain computerized tomography scanning or MRI were included. Patients with head trauma, primary intracranial haemorrhage, or subarachnoid or subdural haemorrhage were excluded. Data were collected by prospective chart review from January 2008 to December 2008. The study protocol was approved by the Regional Bioethics Committee of Isfahan University of Medical Sciences.

\section{Assessment of other variables}

Required information on non-dietary variables such as socio-economic and demographic characteristics (sex, age, education and occupation), past and family medical history, physical activity and smoking status was collected by the use of questionnaires. Height measurement was done in bare feet, weight measurement was done in light clothing and, finally, BMI $\left(\mathrm{kg} / \mathrm{m}^{2}\right)$ was calculated as weight divided by height squared.

\section{Statistical methods}

To ensure normal distribution of variables, we applied the Kolmogrov-Smirnov test. We used two approaches in data analysis: (i) all comparisons were done between cases and controls; and (ii) all comparisons were also performed across tertiles of red meat consumption. Student's $t$ test was used for comparing the means of continuous variables between cases and controls. The $\chi^{2}$ test was applied to the compare distribution of participants between the two groups in terms of categorical variables. Energyadjusted amounts of red meat intake were calculated using the residual method. Then, tertile cut-off points of 
energy-adjusted red meat intake were used for categorizing participants. General characteristics and dietary intakes of participants between cases and controls as well as across tertiles of red meat intake were compared by the use of ANOVA and the $\chi^{2}$ test, where appropriate. All dietary intakes were obtained using general linear models with age and total energy intake as covariates. The logistic regression method was used to examine the associations between red meat consumption and risk of stroke. Estimates are presented in four models. The first model was adjusted for age (continuous), sex (categorical) and total energy intake (continuous). In the second model, we controlled for physical activity (continuous) and smoking (never, ex-smokers, daily smokers). Additional adjustments were made for dietary intakes (including whole and refined grains, vegetables, fruits, low-fat and high-fat dairy, hydrogenated vegetable oil, butter and margarine, nuts and legumes, sugar-sweetened beverages and potato, all as continuous variables) in the third model. Finally, we further adjusted for BMI (continuous), diabetes (yes or no), hypertension (yes or no) and hyperlipidaemia (yes or no) in the fourth model. When the analyses were done across tertiles of red meat intake, the first tertile of red meat consumption was considered as the reference. The overall trend of odds ratios across tertiles of red meat consumption was examined through the use of tertile categories as an ordinal variable in the model. Statistical analyses were performed by using the statistical software package SPSS version $16 \cdot 0$. For all analyses, a value of $P<0.05$ was considered as statistically significant.

\section{Results}

All participants with energy intake less than $3347 \mathrm{~kJ}$ ( $800 \mathrm{kcal})$ or more than $17573 \mathrm{~kJ}$ (4200 kcal) were considered as under- and over-reporters, respectively. Overall, eleven participants could be excluded due to being under- and over-reporters. As in our preliminary analysis the exclusion of this small group of participants did not significantly influence our findings, we decided not to exclude any FFQ.

General characteristics of the study participants based on case and control groups as well as across tertiles of energy-adjusted red meat intake are indicated in Table 1. Participants with stroke were more likely to be male $(60 \%$ v. $47 \%, P<0.05$ ) and older (67.9 (sE 1.0$)$ years $v .61 .5$ (se 0.8$)$ years, $P<0.001$ ) than controls. They had lower weight (69.5 (se 0.9) kg $v .72 .4$ (sE 1.1$) \mathrm{kg}, P<0.05$ ), BMI (25.2 (sE 0.3$) \mathrm{kg} / \mathrm{m}^{2} v .28 .5($ se 1.0$\left.) \mathrm{kg} / \mathrm{m}^{2}, P<0.05\right)$ and were less likely to be obese $(11.3 \% v .29 \cdot 2 \%, P<0.001)$ compared with controls. Hypertension was more prevalent among those with stroke than among controls $(65 \cdot 8 \% v$. $27.2 \%, P<0 \cdot 001)$. In addition, compared with those in the lowest category, participants in the highest category of red meat consumption had higher weight and were more likely

Table 1 General characteristics of the study participants based on case and control groups as well as across tertiles of energy-adjusted red meat intake*; Iranian adults aged $>45$ years, January-December 2008

\begin{tabular}{|c|c|c|c|c|c|c|c|}
\hline & \multicolumn{2}{|c|}{ Cases ( $n$ 195) } & & \multicolumn{2}{|c|}{ Controls ( $n$ 195) } & & \multirow[b]{2}{*}{$P \ddagger$} \\
\hline & Mean & $\mathrm{SE}$ & & Mean & $\mathrm{SE}$ & & \\
\hline Age (years) & $67 \cdot 9$ & 1.0 & & 61.5 & 0.8 & & $<0.001$ \\
\hline Weight $(\mathrm{kg})$ & 69.5 & 0.9 & & $72 \cdot 4$ & $1 \cdot 1$ & & 0.05 \\
\hline BMI $\left(\mathrm{kg} / \mathrm{m}^{2}\right)$ & $25 \cdot 2$ & 0.3 & & 28.4 & 1.0 & & 0.01 \\
\hline Physical activity (MET-min/d) & 4766 & 55.5 & & 3951 & $49 \cdot 1$ & & 0.43 \\
\hline Male (\%) & 60 & - & & 47 & - & & 0.01 \\
\hline Current smoker (\%) & 14 & - & & 6 & - & & 0.01 \\
\hline Obesity† (\%) & 29 & - & & 11 & - & & $<0.001$ \\
\hline Hypertension (\%) & 66 & - & & 27 & - & & $<0.001$ \\
\hline \multirow[t]{4}{*}{ Hyperlipidaemia (\%) } & 22 & - & & 19 & - & & 0.49 \\
\hline & \multicolumn{6}{|c|}{ Tertile of energy-adjusted red meat intake } & \\
\hline & \multicolumn{2}{|c|}{ T1 (lowest) } & \multicolumn{2}{|c|}{$\mathrm{T} 2$} & \multicolumn{2}{|c|}{ T3 (highest) } & \\
\hline & Mean & SE & Mean & SE & Mean & $\mathrm{SE}$ & $P \ddagger$ \\
\hline Age (years) & $62 \cdot 3$ & 1.0 & $64 \cdot 7$ & 1.0 & $62 \cdot 4$ & $1 \cdot 1$ & 0.01 \\
\hline Weight $(\mathrm{kg})$ & $66 \cdot 4$ & 1.1 & 73.3 & $1 \cdot 1$ & 73.6 & 1.3 & $<0.001$ \\
\hline BMI $\left(\mathrm{kg} / \mathrm{m}^{2}\right)$ & 26 & 0.5 & $27 \cdot 4$ & 0.4 & 27.5 & 1.5 & 0.54 \\
\hline Physical activity (MET-min/d) & 4167 & 1173 & 3006 & 566 & 5884 & 848 & 0.08 \\
\hline Male (\%) & 38 & - & 52 & - & 70 & - & $<0.001$ \\
\hline Current smoker (\%) & 5 & - & 8.5 & - & 16 & _- & 0.01 \\
\hline Obesity† (\%) & 19 & - & 27 & - & $15 \cdot 5$ & - & 0.06 \\
\hline
\end{tabular}

MET, metabolic equivalent of task.

${ }^{*}$ Data are means and their standard errors unless indicated otherwise. $P<0.05$ was considered as statistically significant. $\dagger \mathrm{BMI} \geq 30.0 \mathrm{~kg} / \mathrm{m}^{2}$.

¥Obtained by the use of ANOVA or the $x^{2}$ test, where appropriate. 


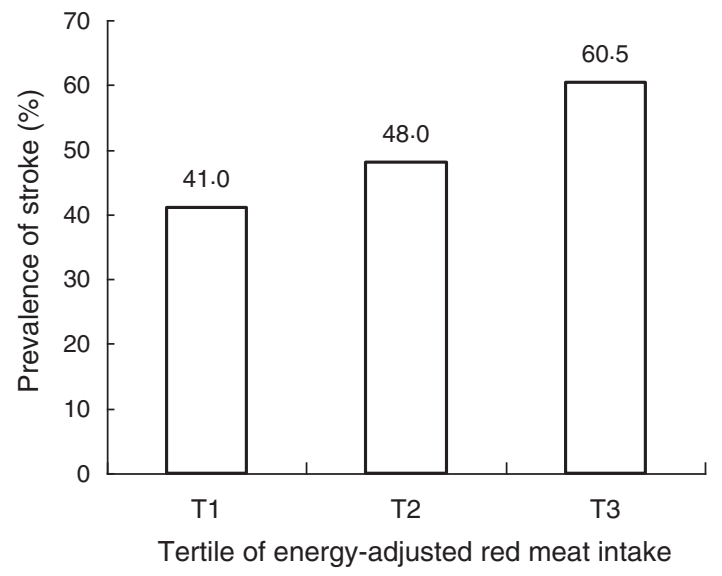

Fig. 1 Prevalence of stroke across tertiles of energy-adjusted red meat intake among group of Iranian adults aged $>45$ years, January-December 2008 ( $P=0.007$, ANOVA) to be male, physically active and current smokers. No significant differences were found in terms of mean BMI and prevalence of obesity across tertiles of red meat intake. The use of medication among cases was listed as follows: aspirin $12.0 \%$, persantine $1.3 \%$, warfarin $5.2 \%$, plavix $1.5 \%$, antihypertensives $26.3 \%$, statins $7.7 \%$, oral hypoglycaemic agents $8 \cdot 8 \%$, insulin $2 \cdot 1 \%$, other drugs $19 \cdot 8 \%$.

The prevalence of stroke across tertiles of energyadjusted red meat intake is shown in Fig. 1. Red meat intake was associated with high prevalence of stroke; such that individuals in the top tertile of red meat intake had higher stroke prevalence compared with those in the bottom tertile $(60.5 \%$ v. $41.0 \%, P=0.007)$.

Dietary intakes of the study participants based on case and control groups as well as across tertiles of energyadjusted red meat consumption are provided in Tables 2 and 3, respectively. Cases with stroke had higher intakes

Table 2 Dietary intakes of the study participants based on case and control groups*; Iranian adults aged $>45$ years, JanuaryDecember 2008

\begin{tabular}{|c|c|c|c|c|c|}
\hline & \multicolumn{2}{|c|}{ Cases ( $n$ 195) } & \multicolumn{2}{|c|}{ Controls ( $n$ 195) } & \multirow[b]{2}{*}{$P+$} \\
\hline & Mean & SD & Mean & SD & \\
\hline Total energy intake $(\mathrm{kJ} / \mathrm{d})$ & 8686 & 4092 & 8828 & 3617 & 0.71 \\
\hline Total energy intake $(\mathrm{kcal} / \mathrm{d})$ & 2076 & 977 & 2110 & 866 & \\
\hline Whole and refined grains $(\mathrm{g} / \mathrm{d})$ & 292 & 168 & 326 & 191 & 0.07 \\
\hline Vegetables $(\mathrm{g} / \mathrm{d})$ & 248 & 172 & 285 & 222 & 0.06 \\
\hline Red and processed meat $(\mathrm{g} / \mathrm{d})$ & 82 & 226 & 57 & 68 & 0.14 \\
\hline Low-fat dairy $(\mathrm{g} / \mathrm{d})$ & 270 & 208 & 340 & 281 & 0.01 \\
\hline High-fat dairy (g/d) & 132 & 208 & 74 & 124 & 0.01 \\
\hline Hydrogenated oil and butter $(\mathrm{g} / \mathrm{d})$ & 19 & 36 & 19 & 30 & 0.93 \\
\hline Sugar-sweetened beverages $(\mathrm{g} / \mathrm{d})$ & 47 & 86 & 47 & 87 & 0.99 \\
\hline Fruits (g/d) & 359 & 413 & 281 & 240 & 0.02 \\
\hline Nuts and legumes $(\mathrm{g} / \mathrm{d})$ & 46 & 48 & 38 & 36 & 0.04 \\
\hline
\end{tabular}

*Data for energy intake are adjusted for age. Data for other dietary variables are adjusted for age and total energy intake. $P<0.05$ was considered as statistically significant.

†Obtained by the use of ANCOVA.

Table 3 Dietary intakes of the study participants across tertiles of energy-adjusted red meat intake*; Iranian adults aged $>45$ years, January-December 2008

\begin{tabular}{|c|c|c|c|c|c|c|c|}
\hline & \multicolumn{6}{|c|}{ Tertile of energy-adjusted red meat intake } & \multirow[b]{3}{*}{$P \dagger$} \\
\hline & \multicolumn{2}{|c|}{ T1 (lowest) } & \multicolumn{2}{|c|}{ T2 } & \multicolumn{2}{|c|}{ T3 (highest) } & \\
\hline & Mean & SD & Mean & SD & Mean & SD & \\
\hline Total energy intake (kJ/d) & 7929 & 3682 & 8238 & 3397 & 10117 & 4155 & $<0.001$ \\
\hline Total energy intake (kcal/d) & 1895 & 880 & 1969 & 812 & 2418 & 993 & \\
\hline Whole and refined grains $(\mathrm{g} / \mathrm{d})$ & 287 & 208 & 307 & 153 & 333 & 174 & $0 \cdot 12$ \\
\hline Vegetables (g/d) & 236 & 198 & 238 & 184 & 325 & 204 & $<0.001$ \\
\hline Red and processed meat $(\mathrm{g} / \mathrm{d})$ & 7 & 4 & 18 & 4 & 89 & 269 & $<0.001$ \\
\hline Low-fat dairy (g/d) & 277 & 231 & 350 & 279 & 292 & 229 & 0.05 \\
\hline High-fat dairy (g/d) & 93 & 180 & 85 & 156 & 130 & 185 & 0.09 \\
\hline Hydrogenated oil and butter (g/d) & 26 & 40 & 21 & 32 & 17 & 30 & 0.15 \\
\hline Sugar-sweetened beverages $(\mathrm{g} / \mathrm{d})$ & 31 & 61 & 44 & 65 & 66 & 118 & 0.01 \\
\hline Fruits $(g / d)$ & 260 & 370 & 306 & 376 & 394 & 317 & 0.01 \\
\hline Nuts and legumes $(\mathrm{g} / \mathrm{d})$ & 33 & 31 & 39 & 36 & 53 & 54 & $<0.001$ \\
\hline
\end{tabular}

*Data for energy intake are adjusted for age. Data for other dietary variables are adjusted for age and total energy intake. $P<0.05$ was considered as statistically significant.

†Obtained by the use of ANCOVA. 
of high-fat dairy, fruits, legumes and nuts, and lower intake of low-fat dairy than the controls. Higher consumption of red meat was associated with higher intakes of energy, fruit and vegetables, low-fat dairy, sugar-sweetened beverages, nuts and legumes, and grains. No significant differences were found for other dietary variables across tertiles of red meat intake.

Crude and multivariable-adjusted odds ratios for stroke based on case and control groups as well as across tertiles of energy-adjusted red meat intake are indicated in Tables 4 and 5, respectively. Red meat intake was significantly associated with the risk of stroke; such that each tertile of red meat intake was related to $90 \%$ increased odds of stroke, after adjustment for confounders (OR $=1.90 ; 95 \%$ CI $1.71,2.85)$. Moreover, in the crude model, individuals in the highest tertile of red meat intake were $119 \%$ more likely to have stroke (OR $=2 \cdot 19$; $95 \%$ CI $1.33,3.60)$ compared with those in the lowest tertile. After controlling for age, sex and total energy intake, the association between red meat consumption and stroke was strengthened $(\mathrm{OR}=2 \cdot 72 ; 95 \% \mathrm{CI} 1.53,4.83)$. This association remained significant even after further controlling for physical activity and smoking as well as dietary intakes.

Table 4 Multivariate-adjusted odds ratios for stroke based on case and control groups*; Iranian adults aged $>45$ years, JanuaryDecember 2008

\begin{tabular}{|c|c|c|c|}
\hline & \multirow{2}{*}{$\frac{\text { Controls }(n \text { 195) }}{\text { OR }}$} & \multicolumn{2}{|c|}{ Cases ( $n$ 195) } \\
\hline & & OR & $95 \% \mathrm{Cl}$ \\
\hline Crude & 1.00 & 1.95 & $1.76,3.25$ \\
\hline Model 1† & 1.00 & 2.08 & $1.66,3 \cdot 11$ \\
\hline Model $2 \ddagger$ & 1.00 & 1.93 & $1.59,2.89$ \\
\hline Model 3§ & 1.00 & $2 \cdot 11$ & $1.63,2.82$ \\
\hline Model 4\| & 1.00 & 1.90 & $1.71,2.85$ \\
\hline
\end{tabular}

${ }^{\star} P<0.05$ was considered as statistically significant.

†Adjusted for age, sex and energy intake.

†Further control for physical activity and smoking.

§Further adjustments for whole and refined grains, vegetables, fruits, low-fat dairy, high-fat dairy, hydrogenated vegetable oil, butter and margarine, nuts and legumes, sugar-sweetened beverages and potato intake.

IAdditionally adjusted for BMI, diabetes, hypertension and hyperlipidaemia.
Additional adjustments for BMI, diabetes, hypertension and hyperlipidaemia did not influence the association significantly; such that participants in the highest tertile of red meat consumption had $151 \%$ greater odds of stroke than those in the lowest tertile $(\mathrm{OR}=2 \cdot 51 ; 95 \%$ CI $1 \cdot 19,5 \cdot 09)$.

\section{Discussion}

In the present case-control study, we found that consumption of red meat was significantly and positively associated with the risk of stroke among a group of Iranian adults. This unfavourable relationship was significant after controlling for different confounding variables, indicating a covariate-independent association. To the best of our knowledge, the current investigation is the first one reporting the association between red meat intake and risk of stroke in a Middle Eastern population.

Stroke is an important cause of mortality and disability globally. Among various dietary components suggested as important modifiable risk factors for stroke, red meat consumption is inconsistently associated with stroke ${ }^{(14-16)}$. While earlier studies have found a positive association between red meat intake and increased risk of stroke, even at a low intake of one serving per day ${ }^{(11,13)}$, red meat intake was not significantly related to incident stroke in other investigations ${ }^{(12,17)}$. Our study indicated that high consumption of red meat is significantly associated with greater odds of stroke. This is in line with a recently published meta-analysis that summarized the existing evidence from epidemiological studies in North America, Europe and Japan. Findings from the meta-analysis revealed a $15 \%$ increased risk of stroke for red and processed meat intake, $9 \%$ for red meat intake and $14 \%$ for processed meat intake ${ }^{(15)}$. Such findings have also been reported in prospective cohort studies. In the Nurses' Health Study and the Health Professionals Follow-Up Study, high consumption of both processed and unprocessed red meat was associated with a greater risk of stroke ${ }^{(20)}$. The positive association between red meat

Table 5 Multivariate-adjusted odds ratios for stroke across tertiles of energy-adjusted red meat intake; Iranian adults aged $>45$ years, January-December 2008

\begin{tabular}{|c|c|c|c|c|c|c|}
\hline & \multirow{2}{*}{$\frac{\mathrm{T} 1<12 \mathrm{~g} / \mathrm{d}}{\mathrm{OR}}$} & \multicolumn{2}{|c|}{$\mathrm{T} 2=12-26 \mathrm{~g} / \mathrm{d}$} & \multicolumn{2}{|c|}{$\mathrm{T} 3>26 \mathrm{~g} / \mathrm{d}$} & \multirow[b]{2}{*}{$P_{\text {trendll }}$} \\
\hline & & OR & $95 \% \mathrm{Cl}$ & OR & $95 \% \mathrm{Cl}$ & \\
\hline Crude & 1.00 & 1.30 & $0.80,2 \cdot 13$ & $2 \cdot 19$ & $1.33,3.60$ & 0.01 \\
\hline Model $1^{*}$ & 1.00 & 1.48 & $0.87,2.52$ & $2 \cdot 72$ & $1.53,4.83$ & 0.01 \\
\hline Model $2 \dagger$ & 1.00 & 1.56 & $0.91,2.68$ & 2.55 & $1.42,4.56$ & 0.01 \\
\hline Model 3‡ & 1.00 & 1.79 & $0.97,3.27$ & $2 \cdot 16$ & $1 \cdot 08,4 \cdot 28$ & 0.02 \\
\hline Model $4 \S$ & 1.00 & 1.68 & $0.95,3.44$ & 2.51 & $1.19,5.09$ & 0.01 \\
\hline
\end{tabular}

${ }^{*}$ Adjusted for age, sex and energy intake.

†Further control for physical activity and smoking.

fFurther adjustments for whole and refined grains, vegetables, fruits, low-fat dairy, high-fat dairy, hydrogenated vegetable oil, butter and margarine, nuts and legumes, sugar-sweetened beverages and potato intake.

$\S$ Additionally adjusted for BMI, diabetes, hypertension and hyperlipidaemia.

IObtained by the use of tertile categories of red meat intake as an ordinal variable in the model. $P<0.05$ was considered as statistically significant. 
intake and stroke has not been indicated in all previous studies. In a meta-analysis in 2010, a null association between red meat intake and risk of stroke was suggested $^{(14)}$. However, in this meta-analysis only three cohort studies, containing small number of stroke cases ( $n$ 2300), were included. A large prospective cohort study conducted among the Japanese population also reported no association between moderate amounts of meat consumption, up to $100 \mathrm{~g} / \mathrm{d}$, and stroke ${ }^{(16)}$. These investigators had included unprocessed poultry in the total meat calculation; however, high intake of poultry was associated with a lower risk of stroke ${ }^{(20)}$. Therefore, lack of a clear description of types of meat might contribute to the discrepancies observed between studies.

In the current study, we had only nineteen cases of haemorrhagic stroke and the other 176 cases had ischaemic stroke. When we excluded cases with haemorrhagic stroke from the analyses, the results strengthened (top $v$. bottom tertile of red meat intake: OR $=2 \cdot 97$; $95 \%$ CI 1.41 , 6.24, $P=0 \cdot 004$ ). Most previous studies have suggested that meat consumption is associated with ischaemic stroke, not with haemorrhagic stroke ${ }^{(15)}$. The different aetiologies of stroke subtypes may be linked differently to risk factors including dietary factors. In addition, the number of patients with haemorrhagic stroke included in most studies was smaller than the number with ischaemic subtype, and thus may limit statistical power and the ability to detect a modest association between meat intake and haemorrhagic stroke. In terms of the types of red meat intake, earlier studies have found stronger associations with processed meats than with unprocessed meats ${ }^{(15,20)}$. In the present study, the red meat category was computed by summing up both processed and unprocessed red meats. We did not include poultry and fish intake in this computation. However, as consumption of processed meats was low (mean: $2.24 \mathrm{~g} / \mathrm{d}$ ), we did not consider that as a separate category.

Several potential mechanisms may explain the adverse effect of red meat consumption on stroke. Red meat is a major source of haem Fe. In an experimental study, the intracellular Fe chelator, desferrioxamine, has been suggested to inhibit inflammation and atherosclerosis, suggesting a potential role of $\mathrm{Fe}$ in atherosclerosis ${ }^{(21)}$. Elevated haem $\mathrm{Fe}$ stores may cause oxidative injury. Epidemiologically, Fe intake has been associated with increased risk of atherosclerosis ${ }^{(22)}$, insulin resistance ${ }^{(23)}$, type 2 diabetes ${ }^{(23)}$ and $\mathrm{CHD}^{(14)}$; all may contribute to the increased risk of stroke. Processed and unprocessed red meats contain high amounts of dietary cholesterol, which has been shown to have adverse effects on serum lipid profiles and subsequently may increase the risk of stroke $^{(22)}$. Furthermore, consumption of red meat was positively associated with risk of hypertension ${ }^{(9)}$, the strongest well-known risk factor for stroke. Na content of processed meats may also explain the positive association observed between meat consumption and risk of stroke ${ }^{(24)}$.
Our findings should be interpreted in the context of potential limitations. Due to the case-control design of the study, causality cannot be inferred. Misclassification of the study participants, as a result of using an FFQ for dietary assessment, could be considered a concern, as it is in most nutritional epidemiology studies. However, the FFQ had previously been validated ${ }^{(19)}$. In addition, due to using an FFQ for assessing intakes, we could not exactly measure individuals' salt intake in order to include it in principal component analysis or make adjustment for it. As we used hospital-based controls in the study, we cannot claim with certainty that controls are representative of healthy adults. In the present study, we did not collect data on cooking methods that might alter health effects of red or processed meats ${ }^{(25)}$. Despite the comprehensive adjustment for confounders in our analyses, some other confounders such as waist circumference and family history of stroke could not be considered due to the lack of data in this regard. In addition, we cannot exclude the possibility of residual confounding. Finally, since we did not perform scanning or imaging in control participants, some controls who might have experienced a silent asymptomatic ischaemic stroke could not be excluded. However, they had no such history, and their examinations were normal.

\section{Conclusion}

In conclusion, we found that consumption of red meat was associated with greater odds of stroke in a group of Iranian adults. This association was independent of potential confounders. Further prospective investigations are needed to confirm this finding.

\section{Acknowledgements}

Financial support: This study was supported by a grant from the Isfahan University of Medical Sciences, Islamic Republic of Iran (no. 187028 IUMS). Financial support for conception, design, data analysis and manuscript drafting comes from the Food Security Research Center, Isfahan University of Medical Sciences, Isfahan, Iran. The Food Security Research Center of Isfahan University of Medical Sciences had no role in the design, analysis or writing of this article. Conflict of interest: None. Authorship: P.S., M.S., A.H.K. and A.E. contributed in the conception, design, statistical analyses and drafting of the manuscript. F.S., A.H.K. and M.S. contributed in data collection. M.B. helped in manuscript drafting. All authors read and approved the final version of the manuscript. A.E. supervised the study. Ethics of human subject participation: All participants provided informed written consent. The study was approved by the Regional Bioethics Committee of Isfahan University of Medical Sciences. 


\section{References}

1. Roger VL, Go AS, Lloyd-Jones DM et al. (2011) Heart disease and stroke statistics - 2011 update: a report from the American Heart Association. Circulation 123, e18-e209.

2. Smith S, Horgan F, Sexton E et al. (2012) The cost of stroke and transient ischaemic attack in Ireland: a prevalencebased estimate. Age Ageing 41, 332-338.

3. Hata J \& Kiyohara Y (2013) Epidemiology of stroke and coronary artery disease in Asia. Circ J 77, 1923-1932.

4. Azarpazhooh MR, Etemadi MM, Donnan GA et al. (2010) Excessive incidence of stroke in Iran: evidence from the Mashhad Stroke Incidence Study (MSIS), a populationbased study of stroke in the Middle East. Stroke $\mathbf{4 1}$, e3-e10.

5. Egeberg R, Olsen A, Christensen J et al. (2013) Associations between red meat and risks for colon and rectal cancer depend on the type of red meat consumed. J Nutr 143, 464-472.

6. Ashaye A, Gaziano J \& Djousse L (2011) Red meat consumption and risk of heart failure in male physicians. Nutr Metab Cardiovasc Dis 21, 941-946.

7. Lajous M, Tondeur L, Fagherazzi G et al. (2012) Processed and unprocessed red meat consumption and incident type 2 diabetes among French women. Diabetes Care 35, 128-130.

8. Azadbakht L \& Esmaillzadeh A (2009) Red meat intake is associated with metabolic syndrome and the plasma C-reactive protein concentration in women. J Nutr 139, 335-339.

9. Tzoulaki I, Brown IJ, Chan Q et al. (2008) Relation of iron and red meat intake to blood pressure: cross sectional epidemiological study. BMJ 337, a258.

10. Larsson SC, Virtamo J \& Wolk A (2011) Red meat consumption and risk of stroke in Swedish men. Am J Clin Nutr 94, 417-421.

11. Larsson SC, Virtamo J \& Wolk A (2011) Red meat consumption and risk of stroke in Swedish women. Stroke $\mathbf{4 2}$, 324-329.

12. Sauvaget C, Nagano J, Allen N et al. (2003) Intake of animal products and stroke mortality in the Hiroshima/Nagasaki Life Span Study. Int J Epidemiol 32, 536-543.

13. He K, Merchant A, Rimm EB et al. (2003) Dietary fat intake and risk of stroke in male US healthcare professionals: 14 year prospective cohort study. BMJ 327, 777-782.
14. Micha R, Wallace SK \& Mozaffarian D (2010) Red and processed meat consumption and risk of incident coronary heart disease, stroke, and diabetes mellitus: a systematic review and meta-analysis. Circulation 121, 2271-2283.

15. Chen GC, Lv DB, Pang Z et al. (2013) Red and processed meat consumption and risk of stroke: a meta-analysis of prospective cohort studies. Eur J Clin Nutr 67, 91-95.

16. Nagao M, Iso H, Yamagishi K et al. (2012) Meat consumption in relation to mortality from cardiovascular disease among Japanese men and women. Eur J Clin Nutr 66, 687-693.

17. Takata Y, Shu XO, Gao YT et al. (2013) Red meat and poultry intakes and risk of total and cause-specific mortality: results from cohort studies of Chinese adults in Shanghai. PLoS One 8, e56963.

18. Esmaillzadeh A \& Azadbakht L (2008) Food intake patterns may explain the high prevalence of cardiovascular risk factors among Iranian women. J Nutr 138, 1469-1475.

19. Esmaillzadeh A, Kimiagar M, Mehrabi Y et al. (2007) Dietary patterns and markers of systemic inflammation among Iranian women. J Nutr 137, 992-998.

20. Bernstein AM, Pan A, Rexrode KM et al. (2012) Dietary protein sources and the risk of stroke in men and women. Stroke 43, 637-644.

21. Zhang WJ, Wei H \& Frei B (2010) The iron chelator, desferrioxamine, reduces inflammation and atherosclerotic lesion development in experimental mice. Exp Biol Med (Maywood) 235, 633-641.

22. Wolff B, Volzke H, Ludemann J et al. (2004) Association between high serum ferritin levels and carotid atherosclerosis in the Study of Health in Pomerania (SHIP). Stroke 35, 453-457.

23. Jiang R, Manson JE, Meigs JB et al. (2004) Body iron stores in relation to risk of type 2 diabetes in apparently healthy women. JAMA 291, 711-717.

24. Strazzullo P, D'Elia L, Kandala NB et al. (2009) Salt intake, stroke, and cardiovascular disease: meta-analysis of prospective studies. BMJ 339, b4567.

25. Bogen KT \& Keating GA (2001) US dietary exposures to heterocyclic amines. J Expo Anal Environ Epidemiol 11, $155-168$. 